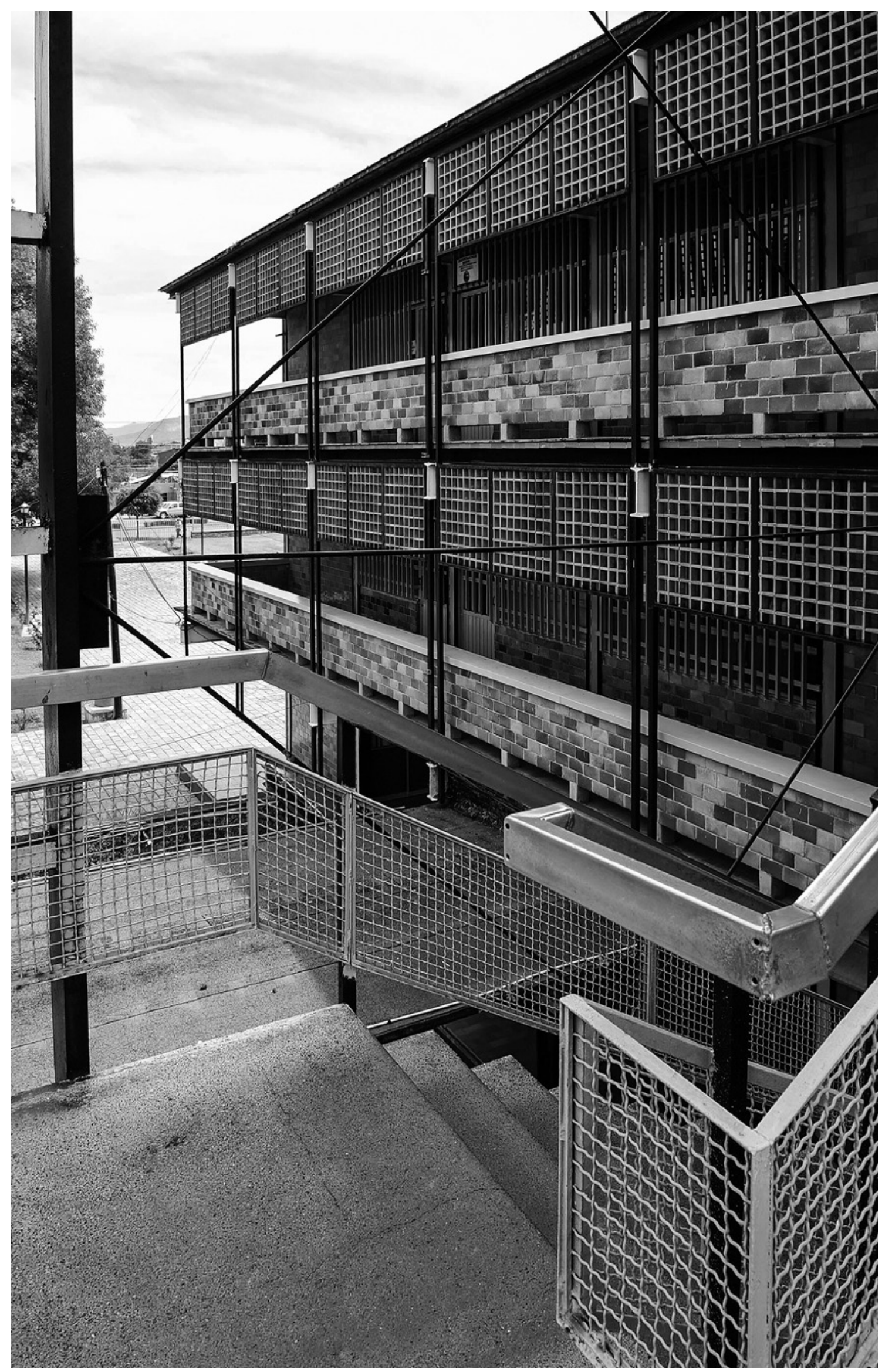

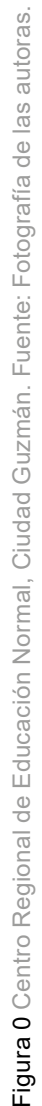



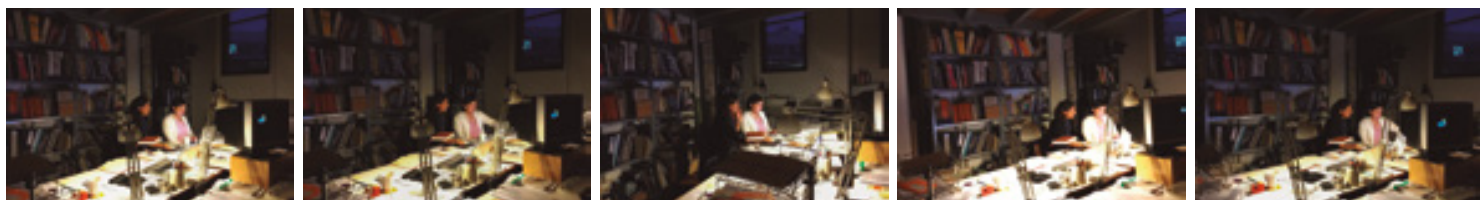

Fotos: Miguel Marcos

\title{
EDUCACIÓN Y ARQUITECTURA: CENTROS REGIONALES DE EDUCACIÓN NORMAL EN MÉXICO, 1960
}

\author{
EDUCATION AND ARCHITECTURE: REGIONAL TEACHER'S COLLEGES (CENTROS \\ REGIONALES DE EDUCACIÓN NORMAL) IN MEXICO, 1960
}

\section{Claudia Rueda Velazquez', Isabela Rentería Cano²}

\section{RESUMEN}

En México, en 1960, se puso en marcha "El plan de once años" promovido por el entonces Secretario de Educación Pública Jaime Torres Bodet. Este programa pretendía resarcir las carencias de la educación básica en México, mediante la construcción de escuelas primarias y la re-estructuración de la formación de los docentes. El programa previó dos sedes pilotos de Centros Regionales de Educación Normal en donde se formarían los maestros desde una visión holística. Ambos centros fueron concebidos como espacios que promoverían la transformación en la cultura y educación regional. Este artículo se centra en estos dos planteles que dan respuesta a una línea de pensamiento. La metodología que se utiliza es un análisis comparativo del programa, ideología y pedagogía del plan de estudios versus el proyecto arquitectónico. Esto lleva a explicar el papel de la arquitectura moderna en el proceso de renovación de la educación en México.

Palabras clave: arquitectura moderna, arquitectura educacional, identidad cultural, historia, regionalismo.

ABSTRACT

In 1960, the "Eleven Year Plan" (El plan de once años), promoted by Jaime Torres Bodet, the Secretary of Public Education at the time, was launched in Mexico. This program intended to compensate for the deficiencies in elementary education through the construction of primary schools and the restructuring of teacher training. The program planned two pilot Regional Teacher's Colleges (Centros Regionales de Educación Normal) where teachers would be trained according to a holistic point of view. Both colleges were conceived as spaces that would promote the transformation of regional education and culture. This article focuses on these two educational establishments, which responded to a certain line of thought. The methodology used for the research involved a comparative analysis of the program, ideology and pedagogy of the plan of study, versus an analysis of the architectural design. This leads to an explanation of the role of modern architecture in the process of renovating education in Mexico.

Keywords: modern architecture, educational architecture, cultural identity, history, regionalism.

Artículo recibido el 14 de agosto de 2017 y aceptado el 20 de diciembre de 2017 DOI: https://doi.org/10.22320/07196466.2017.35.052.10

[1] Centro Universitario Arte Arquitectura y Diseño, Universidad de Guadalajara, Guadalajara, México.claudia.rueda@cuaad.udg.mx [2] Escuela de Arquitectura, La Salle Universidad Ramón Llull, Barcelona, España. isabela@salle.url.edu 


\section{INTRODUCCIÓN}

"Demos a la niñez de nuestro pueblo las aulas y los maestros que necesita. Será la mejor manera de dar un alma -lúcida y vigilante-al progreso de la nación"

(Torres Bodet, 1972: 84)

En el contexto de la historia de la educación en México, a finales del XIX, con el Congreso Higiénico Pedagógico de 1882, surge la preocupación por extender la educación gratuita a amplios sectores de la población, por diseñar espacios específicos para la enseñanza básica y crear escuelas para el aprendizaje del docente -mejor conocidas como "escuelas normales"-. Esta inquietud nace en paralelo a un proyecto de educación y de nación que no terminará de concretarse hasta el s. XX, tras la Revolución Mexicana y, probablemente, con la creación de la Secretaría de Educación Pública en 1921. Como experiencia previa a la búsqueda de un modelo educativo -enseñanza básica, educación de profesores y espacios para la enseñanza- estará La Compañía Lancasteriana ${ }^{3}$, creada en 1822 en la ciudad de México. Aquella compañía fue el primer esfuerzo por fundar un sistema de escuelas gratuitas, formar docentes y diseñar espacios educativos basados en su propio modelo de educación mutual.

La arquitectura para la educación acompaña a dos procesos de enseñanza indisolubles, el de la población y el de los docentes. Esta investigación se centra en las normales "instituciones educativas [...] que giran alrededor de la formación de maestros" (Martínez, 2006:30). En específico, estudia dos Centros Regionales de Educación Normal (CREN), correspondientes a un programa piloto establecido en 1960 para reestructurar la educación docente de enseñanza básica en el marco del "Plan de once años". Dicho proyecto fue encabezado por el pensador, escritor y político Jaime Torres Bodet en su segunda estancia a cargo de la Secretaría de Educación Pública (SEP).

La primera vez que Torres Bodet ocupó el cargo (1943-1946) su labor se concentró en la puesta en marcha de la Campaña de Alfabetización de una población cuyo $50 \%$ no sabía ni leer ni escribir. Tal campaña tenía como prioridad "la construcción de escuelas primarias y normales, reconociendo la poderosa influencia de la educación primaria en el desarrollo cultural del pueblo" (Gual, 1946:3). Para alcanzar el objetivo, en 1944 creó un organismo "descentralizado que habría de encargarse de analizar, plantear e iniciar un programa de construcciones escolares en la República" (González de Cosio, 1958:130) llamado Comité Administrador del Programa Federal de Construcción de Escuelas (CAPFCE). Éste, a través 


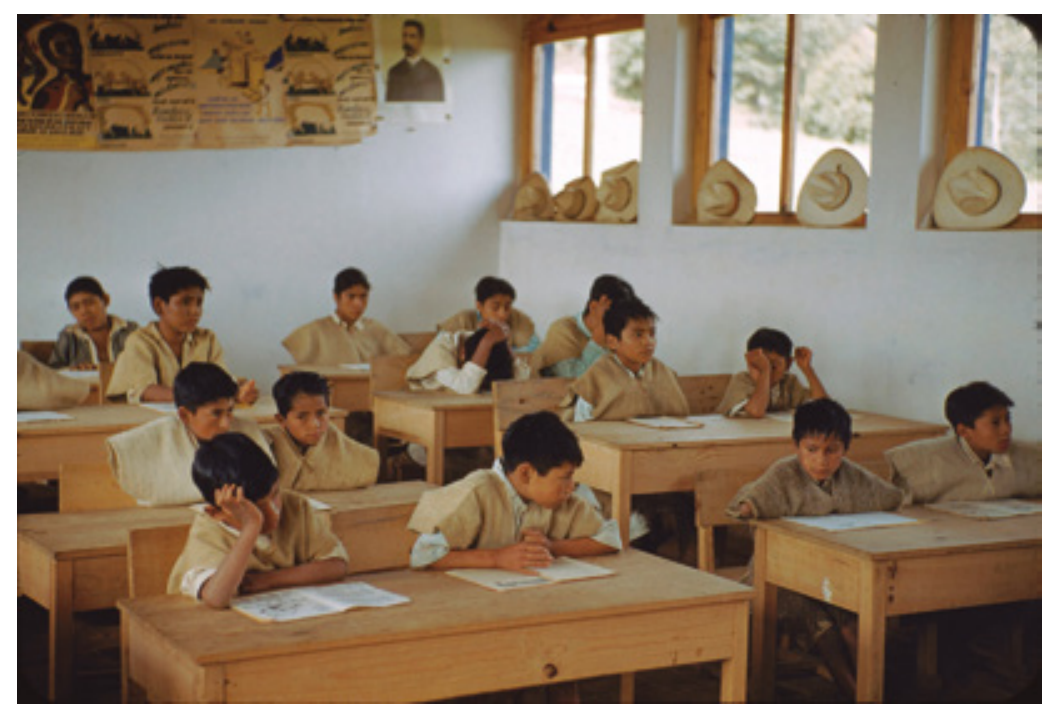

Figura 1 Escuela rural en San Juan Chamula, Chiapas, México (sin fecha). Fuente: Colección Juan Guzmán, Instituto de Investigaciones Estéticas, UNAM.

de su Comisión Técnica y un Jefe de Zona por estado, reunió a arquitectos pioneros de la arquitectura moderna en México ${ }^{4}$, quienes aportaron entonces:

\begin{abstract}
una arquitectura escolar verdaderamente útil, despojándola de lujos y exhibicionismos que no corresponden a nuestras realidades económicas y sociales, y procurando exclusivamente que se satisfagan los requisitos pedagógicos para que nuestras escuelas se adapten a las necesidades del medio natural y social, en las diferentes regiones de nuestro territorio. (Gual, 1946:3)
\end{abstract}

Cuando, en 1958, Torres Bodet retorna como Director de la SEP, el panorama educativo no había variado, el rezago escolar en la población infantil era casi del $50 \%$. Por otra parte, las reformas para las escuelas normalistas que se habían emprendido en su administración anterior no acababan de ajustarse a la realidad educativa del país. Por ello, y tras unos meses de investigación, análisis y planeación, por parte de un grupo de expertos, se pone en marcha un programa titulado "Plan nacional para la expansión y el mejoramiento de la educación primaria en México", mejor conocido como "Plan de once años".

La iniciativa preveía dos acciones básicas: la construcción de escuelas (Figura 1) y la formación de maestros. Para realizar la primera acción el CAPFCE descentraliza sus funciones mediante los gobiernos estatales y nombra como Gerente General al arquitecto Pedro Ramírez
Vázquez. Basado en las experiencias del programa anterior, se promulgó la unificación de los criterios de diseño de las escuelas y se promovió la estandarización como herramienta para hacer una producción más eficiente, capaz de dar soluciones a distintas regiones del país. Así, Ramírez Vázquez y el equipo técnico proponen el aula-casa rural ${ }^{5}$.

La segunda acción era la formación de maestros: la capacitación de los maestros "no titulados" que estaban en servicio y la preparación del suficiente número de docentes para las nuevas aulas; ello se lograría robusteciendo las normales y creando los Centros Regionales de Enseñanza Normal (CREN). Para lanzar el programa piloto, se crearon en 1960 dos centros, uno en Ciudad Guzmán, Jalisco y otro en Iguala, Guerrero, dos poblaciones de menos de 35.000 habitantes: "escogimos esas ciudades porque gran parte de los muchachos que buscaban acogida en los establecimientos capitalinos procedían de Guerrero, de Jalisco, o de entidades cercanas a esos Estados" (Torres Bodet, 1972: 225).

Torres Bodet tuvo la visión de reunir a pioneros y jóvenes arquitectos comprometidos con la modernidad arquitectónica, quienes aportaron desde la arquitectura una respuesta a la ideología y filosofía del plan educativo. La propuesta arquitectónica de este grupo de arquitectos se caracterizaba "por su pertenencia a nuestro tiempo

[4] José Villagrán García, Mario Pani, Enrique Yáñez y José Luis Cuevas; entre los jefes de zona estaban Enrique del Moral, Raúl Cacho, Carlos Le Duc y Pedro Ramírez Vázquez.

[5] El aula-casa rural se diseñó a partir de una ligera armazón metálica prefabricada capaz de recibir muros y techumbres de diversos materiales, adaptándose a las posibilidades de cada comunidad. Recibió el Gran Premio de la XII Trienal de Milán, Italia (1960) y se exportó a 17 países de América Latina. 
y a la región geográfica en que se crea. El regionalismo no puede ignorar a la modernidad, ni ésta abdicar del regionalismo" (Villagrán, 1946:11). Fue en ese contexto en el que se llevan a cabo ambos proyectos, cuya aportación al proceso de renovación de la educación en México se busca explicar en la investigación aquí expuesta.

\section{MÉTODO}

La investigación se desarrolla a partir del método de análisis por comparación. En el estudio de la arquitectura -histórica y de proyecto- resulta indispensable la descomposición de sus partes, para después realizar la síntesis que la explique y la reconstruya. La comparación, según Magda Mària (2017), permite "poner en evidencia aspectos de los proyectos que, de otro modo, no hubiesen surgido. Es como si para estudiar un edificio lo hiciéramos desde la óptica de otro, alumbrando así un punto de vista nuevo". Así, esta propuesta -como se ha indicado previamente- se enfoca en los dos proyectos arquitectónicos de los CREN de Ciudad Guzmán y de Iguala. Es importante señalar que no se personaliza en los arquitectos que conciben la obra, sino en la propia arquitectura.

El análisis se inicia a partir de la estrategia pedagógica del nuevo plan de estudios planteado para las escuelas Normales y la arquitectura construida para apoyarla. Se trata de un estudio desde las intenciones y el contexto del "Plan de once años", en el que, como en otras innovaciones pedagógicas, el espacio donde éstas se implementan se encuentra intrínsecamente vinculado a los nuevos métodos de enseñanza. El examen desvela un proyecto metafórico en relación al plan de estudios, que incide en el entorno urbano, en la comunidad y en la región, como también una contribución desde la modernidad arquitectónica a la renovación educativa y cultural.

Las herramientas para realizar esta comparación han sido las propias del arquitecto: la observación y el re-dibujo. Éstas ayudan a reconocer los principios y criterios arquitectónicos implícitos en las obras y que definen su identidad. Con el fin de desarrollar este proceso, se ha recurrido a un exhaustivo registro documental en publicaciones de la época, imágenes históricas, aerofotos y análisis in situ.

\section{RESULTADOS}

\section{La arquitectura y el plan de educación de enseñanza magisterial}

"El maestro no es exclusivamente un profesional de la educación. Es a lo largo de toda su vida, un ciudadano capacitado para educar" enunciado con el que describía Torres Bodet (1972:80) su concepción de "maestro". Bajo esa premisa y los acuerdos de la Conferencia de Ministros de Educación -Lima, 1956- se ideó el nuevo plan

\begin{tabular}{|c|c|c|c|c|c|c|c|}
\hline \multicolumn{8}{|c|}{ PLAN DE ESTUDIOS DE 3 AÑOS PARA PROFESORES DE EDUCACIÓN PRIMARIA 1960} \\
\hline Semestre & Asignatura & Asignatura & Asignatura & Actividad Anual & Actividad Anual & Actividad Anual & Actividad Anual \\
\hline 1 & $\begin{array}{c}\text { Problemas } \\
\text { económicos, } \\
\text { Sociales y Culturales } \\
\text { de México } \\
6 \text { horas }\end{array}$ & $\begin{array}{l}\text { Lógica } \\
6 \text { horas }\end{array}$ & $\begin{array}{l}\text { Optativa: Español } \\
\text { Superior } \\
\text { Matemáticas } \\
\text { Ciencias Naturales }\end{array}$ & $\begin{array}{c}\text { Talleres, Laboratorios Y } \\
\text { economía Doméstica } \\
3 \text { horas }\end{array}$ & $\begin{array}{l}\text { Actividades } \\
\text { Artísticas } \\
4 \text { horas }\end{array}$ & $\begin{array}{l}\text { Observación } \\
\text { Escolar } \\
6 \text { horas }\end{array}$ & $\begin{array}{c}\text { Educación Física } \\
3 \text { horas }\end{array}$ \\
\hline II & $\begin{array}{c}\text { Antropología Social } \\
\text { y Cultural } \\
6 \text { horas }\end{array}$ & $\begin{array}{l}\text { Ética } \\
6 \text { horas }\end{array}$ & $\begin{array}{c}\text { Psicología general } \\
6 \text { horas }\end{array}$ & $\begin{array}{c}\text { Talleres, Laboratorios y } \\
\text { Economía doméstica } \\
3 \text { horas }\end{array}$ & $\begin{array}{l}\text { Actividades } \\
\text { artísticas } \\
3 \text { horas }\end{array}$ & $\begin{array}{l}\text { Observación } \\
\text { escolar } \\
6 \text { horas }\end{array}$ & $\begin{array}{c}\text { Educación Física } \\
3 \text { horas }\end{array}$ \\
\hline III & $\begin{array}{c}\text { Didáctica general } \\
6 \text { horas }\end{array}$ & $\begin{array}{l}\text { Psicología de la } \\
\text { educación } \\
6 \text { horas }\end{array}$ & $\begin{array}{c}\text { Optativa } \\
\text { (complementaria } \\
\text { o suplementaria) } \\
4 \text { horas }\end{array}$ & $\begin{array}{c}\text { Técnica de la enseñanza } \\
\text { y práctica escolar, taller } \\
\text { de materiales y recursos } \\
\text { didácticos } \\
6 \text { horas }\end{array}$ & $\begin{array}{c}\text { Taller de } \\
\text { Actividades } \\
\text { artísticas Aplicadas } \\
\text { a la escuela Primaria } \\
4 \text { horas }\end{array}$ & $\begin{array}{c}\text { Educación Física } \\
\text { Aplicada a la } \\
\text { escuela Primaria } \\
2 \text { horas }\end{array}$ & \\
\hline IV & $\begin{array}{l}\text { Ciencia de la } \\
\text { educación } \\
6 \text { horas }\end{array}$ & $\begin{array}{l}\text { Educación para la } \\
\text { salud (para varones } \\
\text { educación sanitaria, } \\
\text { para señoritas } \\
\text { puericultura) } \\
6 \text { horas }\end{array}$ & $\begin{array}{c}\text { Optativa } \\
\text { (complementaria } \\
\text { o suplementaria) } \\
4 \text { horas }\end{array}$ & $\begin{array}{c}\text { Técnica de la enseñanza } \\
\text { y práctica escolar, taller } \\
\text { de materiales y recursos } \\
\text { didácticos } \\
6 \text { horas }\end{array}$ & $\begin{array}{c}\text { Taller de actividades } \\
\text { artísticas aplicadas } \\
\text { a la escuela } \\
\text { primaria } \\
4 \text { horas }\end{array}$ & $\begin{array}{l}\text { Educación física } \\
\text { aplicada a la } \\
\text { escuela primaria } \\
2 \text { horas }\end{array}$ & \\
\hline v & $\begin{array}{l}\text { Historia General de } \\
\text { la educación } \\
6 \text { horas }\end{array}$ & $\begin{array}{c}\text { Conocimiento } \\
\text { Del educando } \\
\text { Y psicotécnica } \\
\text { pedagógicas } \\
6 \text { horas }\end{array}$ & $\begin{array}{c}\text { Optativa } \\
\text { (complementaria } \\
\text { o suplementaria) } \\
4 \text { horas }\end{array}$ & $\begin{array}{c}\text { Técnica de la enseñanza } \\
\text { y práctica escolar, taller } \\
\text { de materiales y recursos } \\
\text { didácticos } \\
14 \text { horas }\end{array}$ & $\begin{array}{c}\text { Taller de actividades } \\
\text { Artísticas aplicadas } \\
\text { a la escuela } \\
\text { primaria } \\
3 \text { horas }\end{array}$ & $\begin{array}{c}\text { Educación física } \\
\text { aplicada a la } \\
\text { escuela primaria } \\
2 \text { horas }\end{array}$ & \\
\hline $\mathrm{VI}$ & $\begin{array}{l}\text { Historia de la } \\
\text { educación en } \\
\text { México } \\
6 \text { horas }\end{array}$ & $\begin{array}{l}\text { Legislación, } \\
\text { organización y } \\
\text { administración } \\
\text { escolar } \\
6 \text { horas }\end{array}$ & $\begin{array}{l}\text { Sociología } \\
4 \text { horas }\end{array}$ & $\begin{array}{c}\text { Técnica de la enseñanza } \\
\text { y práctica escolar, taller } \\
\text { de materiales y recursos } \\
\text { didácticos } \\
14 \text { horas }\end{array}$ & $\begin{array}{c}\text { Taller de } \\
\text { Actividades } \\
\text { Artísticas aplicadas } \\
\text { a la escuela } \\
\text { primaria } \\
3 \text { horas }\end{array}$ & & \\
\hline
\end{tabular}


de estudios que se impartiría en los CREN (Tabla 1). Los objetivos fundamentales de aquel plan eran (Martínez, 2006):

Dar al estudiante conocimientos teóricos y prácticos generales y especializados para el trabajo docente.

Inculcar en el alumno altos ideales profesionales y de responsabilidad para realizar su trabajo lleno de renovación espiritual en aras de ser diferente al magisterio en servicio $y$, de tal modo, contribuir al progreso del pueblo mexicano.

Cultivar un sentimiento comunal y nacional.

Constituir a la escuela normal en centro de cultura en su zona de influencia, con la finalidad de incidir en la comunidad en la resolución de sus problemas.

Para responder a estos objetivos, el plan de estudios se estructuraba en tres años con clases teóricas y prácticas. Estas últimas adquirían un protagonismo en el desarrollo conjunto de los CREN y la población que los albergaba; y ello implicaba concebir unos espacios -Escuelas Anexas, campos de cultivo, talleres, oficina servicio comunitario- que los potenciaran.

El servicio comunitario dotaba a los estudiantes de habilidades que les permitirían establecer relaciones con las familias de los niños; así pues, la institución también se implicaba en la vida local con actividades de apoyo a las comunidades rurales. Los CREN (Figura 2) contaban con grandes espacios abiertos en donde se cultivaba; estos espacios no solo tenían una función docente, sino también urbana, pues conectaban los distintos edificios y el conjunto con la población. Con los talleres se confería al docente de unas habilidades enciclopédicas que abarcaban: el trabajo de carpintería, herrería, nociones de asistencia sanitaria o cómo saber nadar, danzar, actuar, cantar, tocar instrumentos musicales; lo cual les dotaba de recursos para incorporarse a cualquier comunidad y aportar una formación adaptada a sus necesidades. Las Escuelas Anexas - primarias y preescolar- tenían un doble objetivo: ser espacios para prácticas profesionales y brindar un servicio a la comunidad. Así, su disposición en el conjunto (Figura 4) se localizaba en un punto estratégico que admitiera esta dualidad; característica que con el paso de los años ha facultado su adaptabilidad a los cambios.

Como resultado del análisis comparativo entre ambos proyectos y su respuesta a los objetivos del plan de estudios y el Plan de once años se destacan tres aspectos básicos de los Centros: su emplazamiento estratégico, su disposición espacial integradora y su sistema constructivo (prefabricación y materiales); todos ellos atienden a la dualidad entre modernidad y región.

Los CREN se localizaron en poblaciones rurales estratégicas en la República mexicana para intentar acompañar el proceso de desarrollo económico, cultural y social de las regiones; se ubicaron en la traza urbana bien relacionados con el núcleo de la población y las comunicaciones (Figura 3). Esto favorecía su papel como motor de la comunidad y de su radio de influencia. El CREN de Ciudad Guzmán (1960-1963) se construyó en los terre-

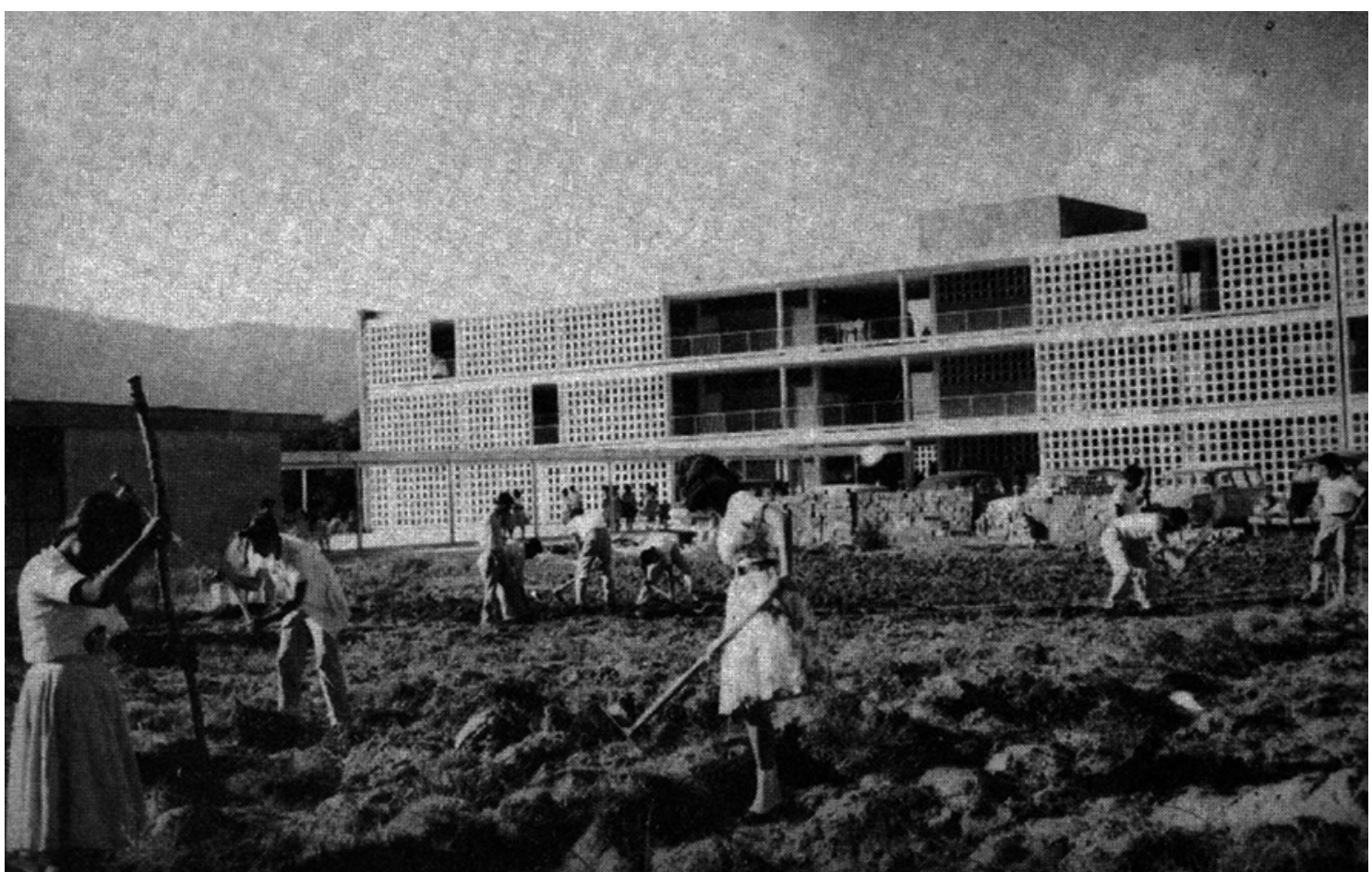

Figura 2 Centro Regional de Educación Normal, Iguala, Guerrero. Clases prácticas de agricultura, 1962. Fuente: Cortesía archivo Guillermo de la Cruz Issa. 

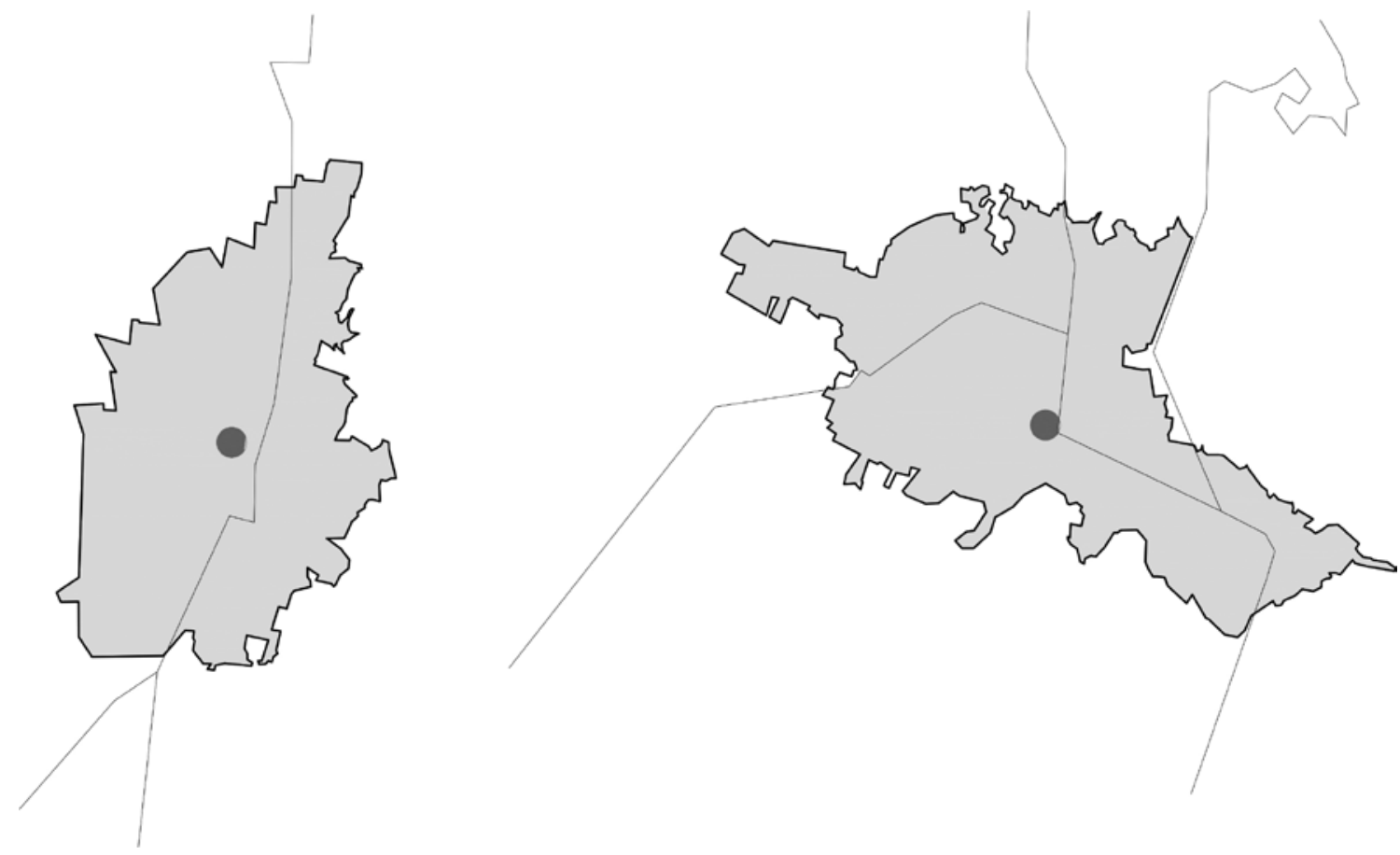

Figura 3 Croquis de ubicación de los Centros Regionales de Educación Normal en relación a la mancha urbana actual. A la izquierda Ciudad Guzmán, Jalisco; a la derecha, Iguala, Guerrero. Se observa su ubicación estratégica en relación a las vías de comunicación de la región. Fuente: Elaboración de las autoras.

01 Acceso

07 Instalaciones deportivas (sección de recreación)

02 Administración

08 Escuela primaria (escuela anexa)
09 Escuela primaria (escuela anexa)

03 Sección enseñanza teórica

04 Talleres (enseñanza práctica)

05 Biblioteca (sección difusión cultural)

10 Centro de atención múltiple
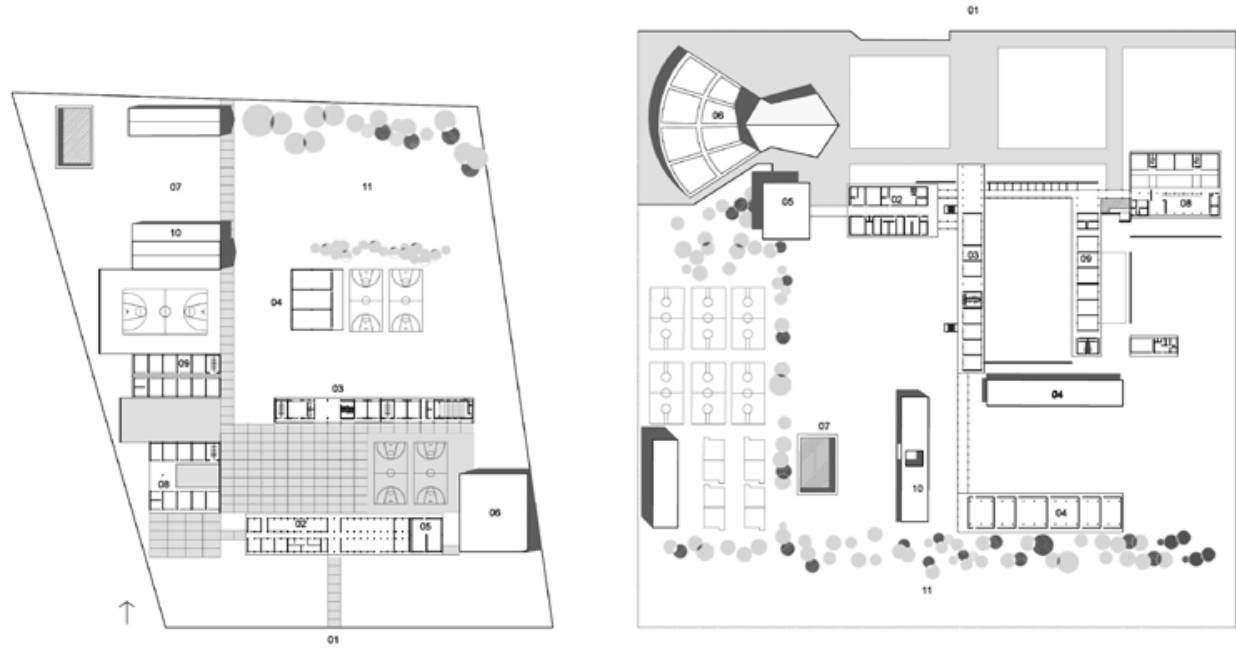

Figura 4 Hipótesis de las plantas de conjunto en su estado original de acuerdo con distintas fuentes: edificios originales representados en plantas bajas y edificios añadidos posteriores a 1960, representados en planta cubierta. A la izquierda Iguala, Guerrero; a la derecha, Ciudad Guzmán, Jalisco. Fuente: Elaboración de las autoras. 
nos próximos a la estación de ferrocarril, en la carretera que la une con el centro urbano y otras vías. El terreno, en forma de cuadrado, tiene una superficie de $16 \mathrm{ha}$. El CREN de Iguala (1960-1963) se ubica a un costado de la carretera federal México-Cuernavaca-Iguala en un solar de forma romboidal de aproximadamente $4.5 \mathrm{ha}$. Ambos terrenos tenían en común su planicie y de fondo el Volcán Nevado y el cerro del Tehuehue, respectivamente. Tras la construcción de los dos centros, alrededor de ellos se formaron barrios; en el caso de Ciudad Guzmán, el de maestros y toda una serie de infraestructuras y servicios que incentivaron la economía y el desarrollo del lugar.

\section{Disposición espacial}

El conjunto arquitectónico de ambos planteles se relaciona con las secciones del plan de estudios ${ }^{6}$ (De Alba, 1963:41) y se despliega en distintas piezas volumétricas según un esquema moderno funcionalista, en el que cada una de ellas alberga un uso. La pieza principal la forma el núcleo de enseñanza teórica (Figura 4).

En Ciudad Guzmán, el edificio principal es un paralelepípedo de tres alturas que ocupa el eje del conjunto y del que se ramifican las distintas edificaciones de una planta que se extienden horizontalmente por el terreno. Por el noreste se sitúa la Escuela Anexa, por el sur, los talleres y el núcleo de enseñanza experimental y, por el noroeste, la administración, de la cual se anexan biblioteca, el auditorio y anfiteatro. Estos últimos, además del edificio principal, destacan por su volumetría singular y por su papel, como detonante cultural en la comunidad. Auditorio y anfiteatro comparten escenario lo que permite presenciar una misma puesta en escena desde ambos espacios.

La disposición del proyecto -haciendo un símil con el plan de estudios- se entiende como un conjunto de ramificaciones que componen una formación compuesta, variada pero unitaria. Todos los volúmenes están conectados con pasarelas que protegen del sol (Figura 8), y entre ellos se va generando espacios abiertos, jardines, explanadas y plazoletas. El terreno queda delimitado y ordenado, y en él se despliegan también las canchas, las pistas deportivas y los campos para la práctica de la agricultura. Las intersecciones con los edificios dan lugar a vaciados y solapes inteligentemente resueltos. El vaciado dota de permeabilidad a los edificios y les da continuidad espacial que facilita los recorridos y el acceso. El predominio de la línea horizontal aproxima la escala del conjunto a las personas.

El edificio principal de aulas se posiciona perpendicularmente a la calle y vuela sobre el muro de piedra paralelo a la misma calle, señalando así el acceso al recinto y sobrepasando los límites del cerramiento (Figura 5). Mientras todos los edificios procuran su iluminación norte, y se protegen del sur, el volumen central tiene una orientación este-oeste, debido a que por su altura se vería de otra forma más expuesto a los vientos dominantes del sur que arrastran tierra desde poniente (Mendoza, 2004). Esta orientación provoca la necesidad de protección del sol y es el motivo por el que se disponen las celosías que protegen las fachadas, uno de los elementos plásticos más interesantes de su arquitectura.

En el CREN de Iguala (Figura 4) el programa también se dispone en distintos bloques. El edificio de aulas teóricas ocupa la posición central y destaca del resto del conjunto -como ocurre en el complejo de Ciudad Guzmán- por su altura de tres plantas. En este caso, la envolvente del edificio de aulas se protege de la orientación sur mediante una galería cerrada, compuesta por paneles de celosías de hormigón que le confieren una fachada de gran abstracción. El resto de los edificios - de una planta- se extienden horizontalmente, en ocasiones perpendicular o paralelamente al edificio central, $y$ dan forma a los espacios abiertos, explanadas, jardines $y$ espacios deportivos (Figura 6)

La colocación de los edificios principales es paralela a la calle y el acceso se realiza perpendicularmente a los edificios: por las partes diáfanas de las plantas bajas. Primero se atraviesa el edificio de una planta de administración y biblioteca, y bajo una pasarela que cruza la explanada principal se llega al edificio de aulas. Al igual que en Ciudad Guzmán, la accesibilidad y circulación son fluidas, y procuran que el conjunto se acerque a la comunidad donde se inserta. El polideportivo cerrado se posiciona junto al edificio de administración a la derecha del eje de acceso.

\section{Prefabricación y materiales}

Los lineamientos generales del CAPFCE, tanto para escuelas primarias como para los CREN, promovieron un proyecto dual, entre lo industrial y lo artesanal. E empleo de materiales prefabricados tenía la bondad de acortar los tiempos y abaratar los costos; y la convivencia de éstos con técnicas constructivas del lugar permitió una "variedad de envolventes utilizados para los distintos climas, y de materiales y técnicas constructivas locales, con las que se complementó el marco prefabricado de acero, distribuido masivamente por todo el país" (Arañó, 2011:317). Prueba de ello, son estos dos proyectos, con soluciones constructivas y de control climático (celosías) mixtas.

EI CREN, en Ciudad Guzmán, de Salvador de Alba, se distingue por el fino ensamblaje de sus elementos constructivos (Figuras 7 y 8 ). El conjunto está construido a base de elementos de acero, tanto en el sistema estructural como en su sistema de fachada corrida, y de perfiles que soportan los cerramientos. Los pilares son perfiles de alma abierta entre los que se insertan las vigas, como en un ensamblaje. La estructura se cierra con superficies de fábrica de piezas cerámica, con técnicas locales, colocadas en paredes, bóvedas del forjado y paneles celosía. Todo queda expuesto, sin recubrir. 


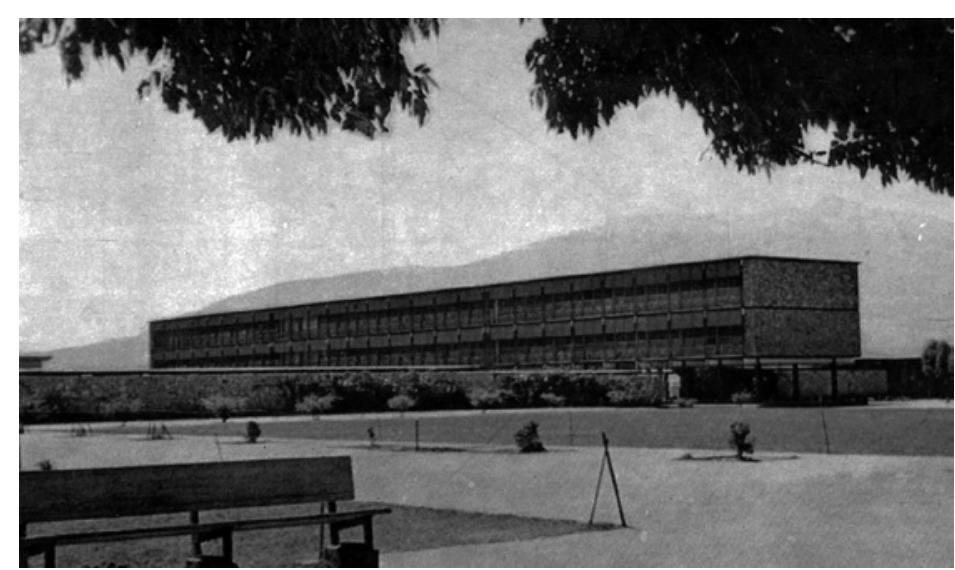

Figura 5 Centro Regional de Educación Normal, Ciudad Guzmán, Jalisco, 1960 Fuente: Cortesía Archivo histórico CREN, Ciudad Guzmán.

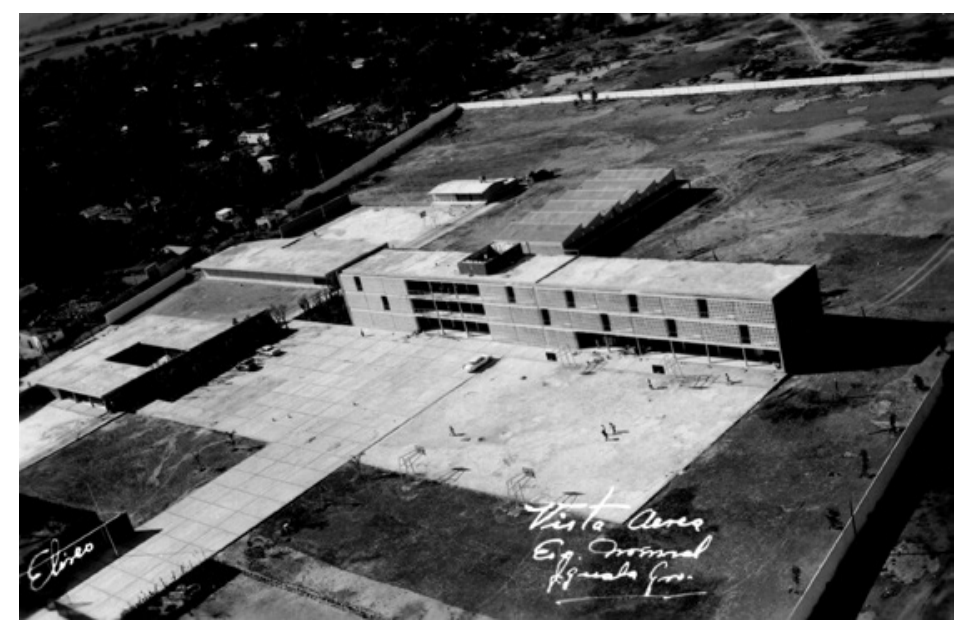

Figura 6 Centro Regional de Educación Normal, Iguala, Guerrero, 1960. Fuente: Fotografía Eliseo; Cortesía Archivo Guillermo de la Cruz Issa.

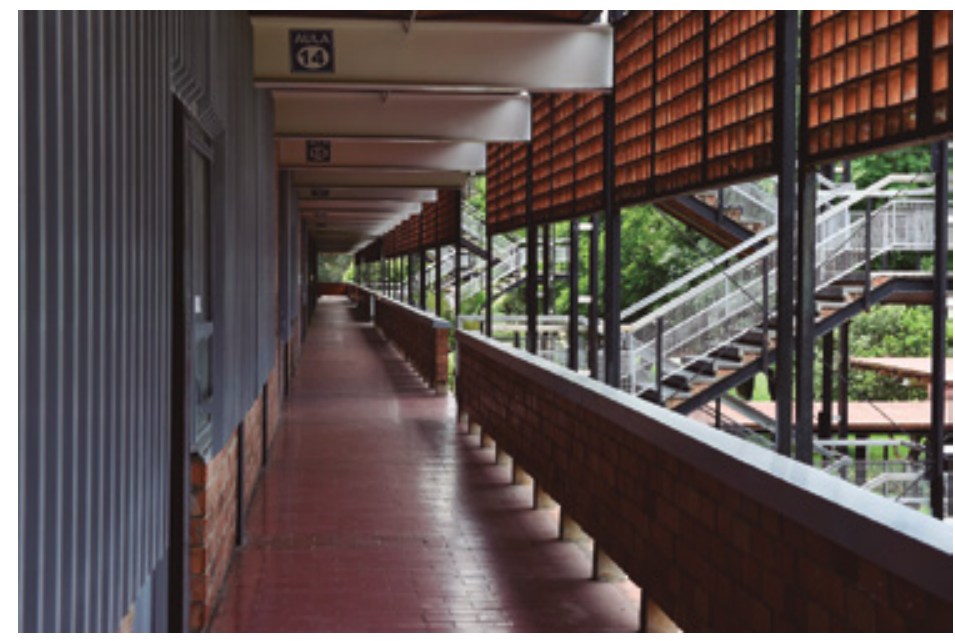

Figura 7 Pasillo del edificio principal del Centro Regional de Ciudad Guzmán. Fuente: Fotografía de las autoras. 


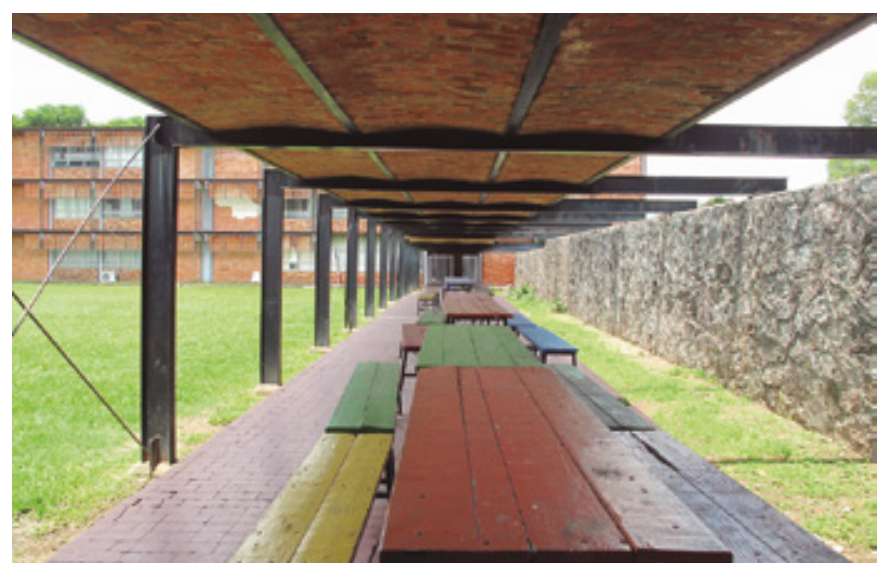

Figura 8 Pasarela de conexión entre edificio principal y Escuela Anexa, Centro Regional de Ciudad Guzmán. Fuente: Fotografía de las autoras.

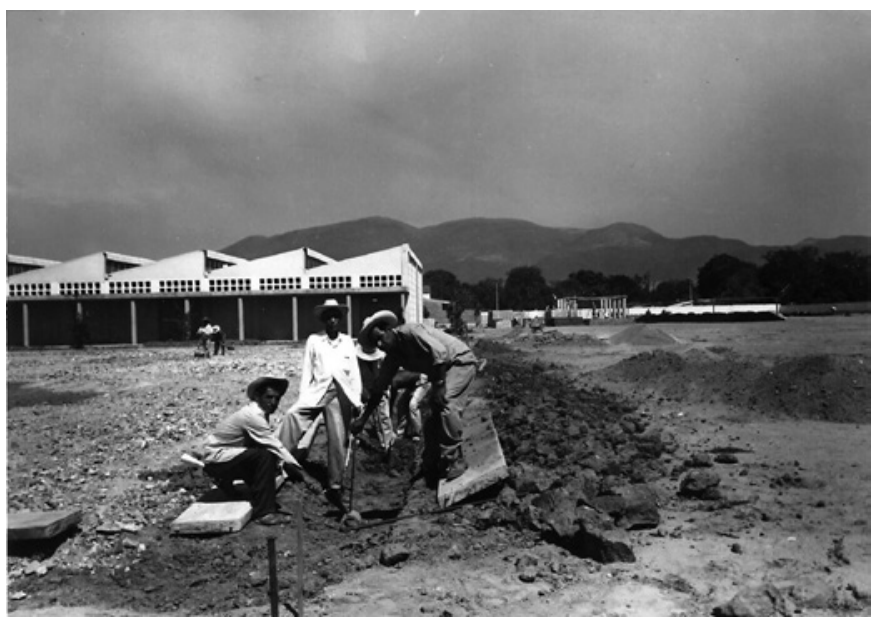

Figura $9 \mathrm{Al}$ fondo, talleres de prácticas y, al frente, alumnos construyendo el área deportiva en el Centro Regional de Educación Normal, Iguala, Guerrero, 1960. Fuente: Cortesía Archivo Guillermo de la Cruz Issa.

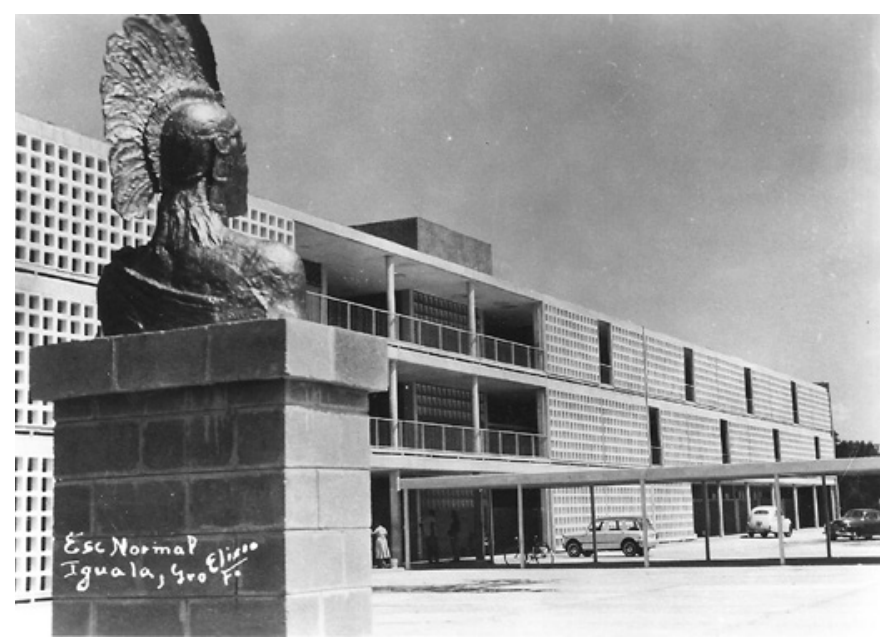

Figura 10 Edificio principal en el Centro Regional de Educación Normal, Iguala Guerrero, 1960. Fuente: Fotografía Eliseo; Cortesía Archivo Guillermo de la Cruz Issa. 


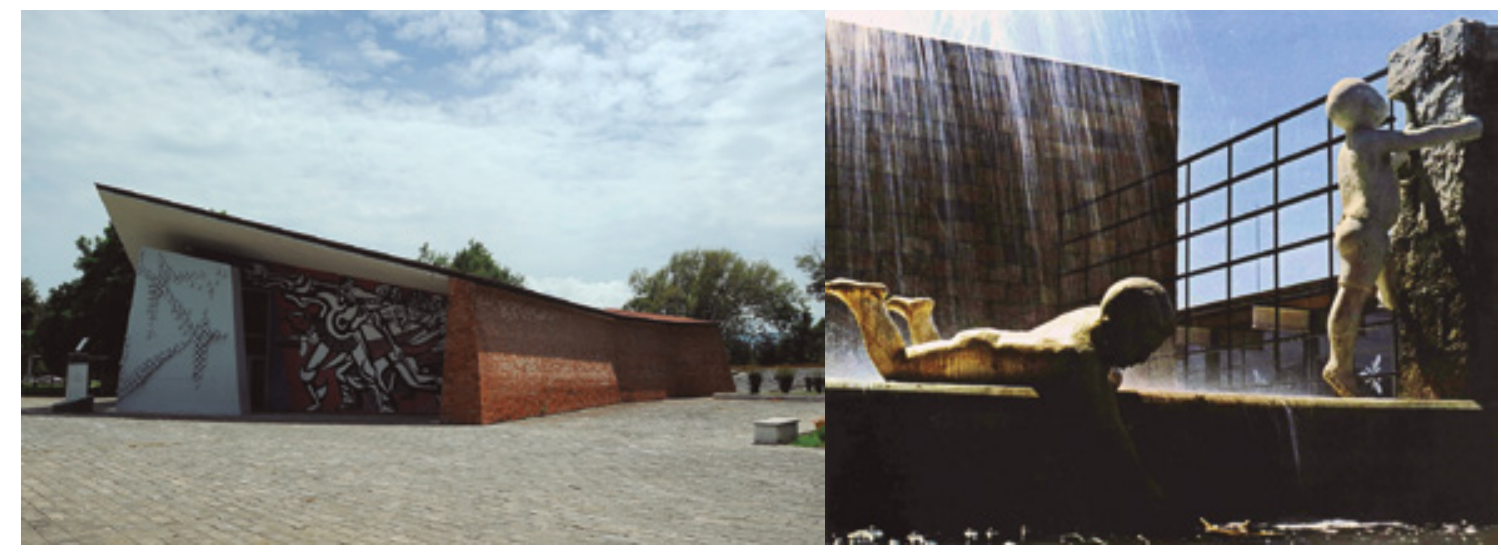

Figura 11 Intervención plástica en el Centro Regional de Educación Normal, Ciudad Guzmán. "La pastora" (integración plástica en el auditorio) y la fuente de "Los niños" en el ingreso a la Escuela Anexa, obras del Mtro. Tijerino. El mural en el auditorio Mtro. Delgadillo en colaboración estudiantes. Fuente: Izquierda: fotografía de las autoras. Derecha: Cortesía Archivo histórico CREN, Ciudad Guzmán.

Las diferentes capas que rodean las aulas del edificio principal acondicionan el espacio climáticamente: las celosías de las dos fachadas ocupan el tercio superior de cada piso y evitan así la entrada del sol directo a las aulas, sin cerrar las vistas. Tienen un aspecto liviano: cada celosía posee tres módulos dentro de bastidores metálicos pintados en negro, sujetados por perfiles tensores que cuelgan de las vigas principales (Figura 7). Éstas vuelan hasta la fachada y están pintadas de blanco; cada pieza se destaca y el ensamblado se hace, de este modo, más aparente. La barandilla de obra de cerámica vidriada también parece un elemento autónomo, no coincide con el plano de fachada y flota sobre soportes.

En Iguala, la implicación de la comunidad (Figura 9) se reforzó no solo mediante la utilización de técnicas locales que completaban los elementos de la estructura, sino con la mano de obra de los propios estudiantes: "se fue construyendo paulatinamente y algunos alumnos varones se alquilaban como peones en las vacaciones para trabajar en las construcciones pendientes y así apoyarse con sus gastos personales" (De la Cruz, 2010:15).

El conjunto se construyó con elementos prefabricados de acero en estructura y de concreto armado en losas de forjado y celosías (Figura 10). Las paredes y cerramientos se elaboraron con ladrillo. En la fachada sur, el cerramiento queda remetido para dejar el pasillo de acceso abierto y se protege de suelo a techo por una celosía que evita el asoleamiento directo del interior y genera una galería colchón de acondicionamiento climático. Las vistas hacia el exterior se disponen en aperturas intercaladas entre los paneles de celosía. La fachada se compone con las líneas horizontales de las losas y los paneles de celosías que apoyan en ellas.

Los dos ejemplos que aquí se han analizado se caracterizan por combinar sistemas tradicionales con tecnologías de vanguardia, tanto en la construcción como en el amueblamiento interior. En ambas escuelas, los elementos constructivos repetidos responden a su función práctica y desde la expresión veraz o explícita del sistema utilizado, dignifican el conjunto con una expresión abstracta de gran plasticidad. Las dos obras son exponentes de virtudes constructivas, económicas y sociales; lugares donde los profesionales -con una buena dosis de experiencia y sensibilidad-integran, asimismo, las restricciones económicas con el potencial de la mano de obra y materiales locales, y devienen de un programa piloto de progreso y del compromiso de la arquitectura con los espacios para la educación.

\section{CONCLUSIONES}

El "Plan de once años" planteó uno de los grandes retos al que se enfrentaba el México moderno: lograr que la educación llegara a cada una de las regiones del país. En este reto, la concepción arquitectónica cumplía un papel fundamental de acompañar el proceso de renovación educativa. Los proyectos arquitectónicos del CREN de Ciudad Guzmán e Iguala respondieron, no sólo -a través del programa arquitectónico y espacial- a la reforma educativa para los profesores, sino además, secundaron las líneas de pensamiento de la época. Su arquitectura destaca por la capacidad de potenciar las actividades humanas que sostenía mediante su adecuación al lugar y al tiempo: realiza una conciliación estratégica con el plan de estudios y un maridaje entre los objetivos de éste y las cualidades espaciales. Los siguientes aspectos refuerzan esta conclusión:

Proyecto integrador. Se ha visto como su implantación expande la actividad a la periferia y construye espacios para la ciudad. La arquitectura de los dos centros está pegada al terreno, literal y figurativamente, y en ella se prioriza la racionalización versus el papel simbólico de la institución. En la implantación del proyecto se concreta "la contribución al progreso del pueblo", el fomentar la cultura y educación en la comunidad a través no sólo de las actividades culturales en el auditorio, foro y espacios recreativos, sino 
como telón de expresiones culturales como los murales, esculturas ${ }^{7}$ (Figura 11). Las implantaciones en el territorio, de alguna forma, marcaron la llegada de la modernidad no solo en términos arquitectónicos sino de desarrollo.

Flexibilidad de la traza y la edificación. Una educación percibida como formación global se acompañó con una propuesta arquitectónica planteada como un complejo no cerrado, permeable figurativa y espacialmente. En un símil con el plan de estudios -en el que la pieza principal es el núcleo teórico del que se desprenden las demás materias- la disposición espacial de ambos conjuntos sigue el esquema de un edificio principal (Figuras 5 y 6 ) del que se extienden las partes. Se realiza sobre una traza flexible de líneas perpendiculares donde se ubican edificios y recorridos, y permite un crecimiento opcional.

Estandarización y adaptación. Sin lujos ni exhibiciones, con orden sin jerarquías, de mano de la modernidad, la arquitectura potenció una visión renovadora tanto en su composición como en su sistema de construcción: útil, eficiente, polivalente, tal y como se debía formar al profesor, con requisitos pedagógicos adaptados al medio y cercanos a la gente. La estandarización en el programa educativo permitía llegar a todas las poblaciones de México y adaptarlo a las mismas. De igual forma sucedía con la arquitectura, la estandarización y prefabricación constructiva permitía llegar a los lugares más alejados; y la mano de obra local aportaba el conocimiento constructivo y los materiales del lugar que la mimetizaban con el entorno.

Pareciera que aquella frase de José Villagrán donde manifestaba que el regionalismo no podía ignorar a la modernidad y viceversa, será el sentido por el cual ambos proyectos en Ciudad Guzmán e Iguala validan su atemporalidad y vigencia, y los hace resistentes a modificaciones y evoluciones de los modelos educativos.

Si bien, los dos Centros Regionales de Educación Normal (CREN) comparten programas y criterios de orientación, disposición, acondicionamiento y construcción, el ensamblaje y detalles son aportaciones particulares de la arquitectura en cada uno de los casos. La arquitectura de las dos escuelas resulta lacónica, ordenada según una lógica constructiva que transmite su belleza desde su pragmatismo y el buen hacer, y su expresión más lírica surge de una combinación mágica entre ritmo, mezcla de técnicas y materiales, colores y el tratamiento sutil y funcional de las superficies. Su arquitectura y la generosidad de sus espacios aportan ese plus necesario para acometer el objetivo -más allá de su función- de que las comunidades adquiriesen "la noción exacta del valor de la enseñanza y sentir la dignidad que exige el marco en el que se formarán los niños y vivirán los maestros" (Torres Bodet, 1963:72).

[7] En el auditorio hay una intervención plástica titulada "La pastora", en la explanada de acceso, la fuente "Ascender" y en el ingreso a la Escuela Anexa, la fuente de "Los niños"; todas obras del Mtro. Tijerino. (SOLANO REYES, Eliazar. Entrevista realizada por las autoras el 8 de agosto de 2017).

\section{REFERENCIAS BIBLIOGRÁFICAS}

ARAÑÓ, Axel. Prefabricación y estandarización. En: ARAÑO, Axel (ed.), Arquitectura Escolar. SEP 90 años. México: Secretaría de Educación Pública, Consejo Nacional para la Cultura y las Artes y Fragonard 70 S.C., 2011.

CRUZVILLEGAS, Abraham. El escarabajo del Arte Contemporáneo. El País Semanal [en línea]. 2015, 16 de septiembre. [Consulta 2 de agosto 2017]. Disponible en: https://elpais.com/elpais/2015/09/11/ eps/1441988068_088281.html

DE ALBA, Salvador. El uso de las estructuras del sistema aula casa rural en diversas aplicaciones escolares. Arquitectos de México, 1963, tomo IV, n 17, pp. 41-43.

DE LA CRUZ ISSA, Guillermo. Centro Regional de Educación Normal, Iguala de la Independencia, 50 aniversario. Memorias 1960-2010. Guerrero, 2010.

GONZÁLEZ DE COSIO, Francisco. Construcción de Escuelas. Laboradas por el CAPFCE 1953-1958. Arquitectura México, 1958, tomo XIV, n 63, pp. 123-134.

GUAL VIDAL, Manuel. Al lector. En: CAPFCE (coord.), Comité Administrador del Programa Federal de construcción de Escuelas y su obra. Memoria de la Primera Planeación, Proyección y Construcciones Escolares de la República Mexicana 1944, 1945,1946. México D.F.: CAPFCE, 1946, pp. 3-5

MÀRIA SERRANO, Magda. Musqueda Silvia. Arquitectura Comparada. Clase inaugural del curso Arquitectura Comparada en el marco del Máster MBArch, UPC, 17 de febrero 2017.

MARTÍNEZ LORENZANA, Francisco (coord.). El pasado y el presente de una institución educativa: EI CREN de Iguala, Gro. Estudio de caso, Centro Regional de Educación Normal de Iguala, Gro. Iguala, Guerrero: Área de Investigación educativa, 2006-2007.

MENDOZA, Héctor. Aportación de la Escuela Tapatía. Edificios de Carácter Colectivo de 1957 a 1968 en el Estado de Jalisco. Tesis doctoral inédita, Universidad Politécnica de Cataluña, 2004.

ROTH, Alfred. The New school. London: Zurich Gisberger, 1950.

TORRES BODET, Jaime. Écoles Préfabriqués, Mexique. Architecture D'Aujourd'hui, 1963, no 109, pp.7280.

TORRES BODET, Jaime. Memorias. La tierra prometida. México: Editorial Porrúa, 1972.

VILLAGRÁN GARCÍA, José. El Comité Administrador del Programa Federal de construcción de Escuelas y su obra. En: CAPFCE (coord.), Comité Administrador del Programa Federal de construcción de Escuelas y su obra. Memoria de la Primera Planeación, Proyección y Construcciones Escolares de la República Mexicana 1944, 1945, 1946. México D.F.: CAPFCE, 1946, pp. 1114. 